\title{
SPLITTINGS OF SURFACES
}

\author{
RICHARD K. SKORA
}

Let $\Gamma$ be a finitely generated group and let $\mathcal{D}_{n}(\Gamma)$ be the space of discrete, faithful representations of $\Gamma$ into the group of isometries of hyperbolic $n$-space divided out by conjugation. William P. Thurston [Th1] studied this space in the cases where $\Gamma$ was the fundamental group of a surface or 3-manifold. Let $F$ be a closed surface of negative Euler characteristic. For convenience fix a hyperbolic structure on F. Thurston constructed a compactification of $\mathcal{D}_{2}\left(\pi_{1} F\right)$ which is homeomorphic to the closed ball of dimension $-3 \chi(F)$. The interior is identified with $\mathcal{D}_{2}\left(\pi_{1} F\right)$. Thurston showed that the boundary of the ball is identified with the space of $\mathbf{R}$-projective measured geodesic laminations on $F$ [Th1].

John W. Morgan and Peter B. Shalen [Mo-Sh1], [Mo1] showed that for any $\Gamma$ and any $n$ the space $\mathcal{D}_{n}(\Gamma)$ admits a compactification where each ideal point corresponds to a small action by isometries of $\Gamma$ on an $\mathbf{R}$-tree.

An $\mathbf{R}$-tree is a metric space $(T, d)$, such that any two distinct points are joined by a unique arc and every arc is isometric to a segment in $\mathbf{R}$. A group is small if it does not contain a free group of rank two. An action is small if the stabilizer of each non-degenerate arc is small. If $F$ is any connected 2-manifold other than a torus, then the small subgroups of $\pi_{1}(F)$ are cyclic.

In the special case $\Gamma=\pi_{1} F$ and $n=2$, Morgan and Shalen argue in terms of $\mathbf{R}$-trees to show that their boundary is naturally identified with the space of projective measured geodesic laminations on $F$. This recovers Thurston's result. Different proofs of Thurston's result from the point of view of $\mathbf{R}$-trees appeared later in [Mo-Ot] and [Sk2].

Shalen $[\mathrm{Sh}]$ posed several problems stemming from their work. In particular, he advertised that the most direct proof of the above would be via a positive answer to the following question. Is every small action of the fundamental group of a closed surface on an $\mathbf{R}$-tree dual (see Section 2) to a measured geodesic lamination? This question is answered by the following theorem which was announced in [Sk1].

Theorem 3.2. Let $F$ be a hyperbolic surface of finite area and let $\pi_{1} F \times T \rightarrow T$ be a non-trivial action on an $\mathbf{R}$-tree, such that $\pi_{1} E$ fixes a point for each cusp $E$ of $F$. Then $\pi_{1} F \times T \rightarrow T$ is small if and only if it is dual to a measured compact geodesic lamination.

There have been partial results towards the above theorem. Morgan-Shalen [Mo-Sh1], using the techniques of John Stallings, proved it in the case that the

Received by the editors November 13, 1989 and, in revised form, March 23, 1992, March 15, 1994, and May 15, 1995.

1991 Mathematics Subject Classification. Primary 05C38, 15A15; Secondary 05A15, 15A18.

Key words and phrases. R-tree, surface, hyperbolic space, splitting.

Supported in part by a SUNY Research Development Grant and an NSF Postdoctoral Research Fellowship. 
$\mathbf{R}$-tree is homeomorphic to a simplicial tree. Morgan and Jean-Pierre Otal [Mo-Ot] proved it under an additional geometric hypothesis (cf. [Sk2]), and Henri Gillet and Shalen [Gi-Sh] proved it in the case that the action has rank 1 or 2 (see Section 5 for the definition of $r a n k$ ).

Here is an outline of the proof of the main theorem. If $\pi_{1} F \times T \rightarrow T$ is dual to a measured compact geodesic lamination, then it is obvious and well known that the the action is small. Conversely, suppose $\pi_{1} F \times T \rightarrow T$ is a small action. The first step is a result of Morgan and Otal [Mo-Ot], of which a simplified proof was given by Allen Hatcher [Ha]. This result gives an action $\pi_{1} F \times R \rightarrow R$ which is dual to a measured compact geodesic lamination $(\mathcal{L}, \mu)$, and a $\pi_{1} F$-equivalent morphism $\phi: R \rightarrow T$.

Using the hypothesis that $\pi_{1} F \times T \rightarrow T$ is small, one needs to show that $\phi$ is an isometry. The proof of this begins with the standard observation that the firstreturn map for a measured lamination $(\mathcal{L}, \mu)$ is an interval exchange. In terms of the dual tree $R$ to $(\mathcal{L}, \mu)$, this means that a short segment $I$ in $R$ can be covered by nonoverlapping intervals $I_{1}, \ldots, I_{n}$ to which there are associated elements $g_{1}, \ldots, g_{n} \in$ $\pi_{1} F$ such that $g_{1}\left(I_{1}\right), \ldots, g_{n}\left(I_{n}\right)$ are again non-overlapping intervals in $I$. In fact, if we identify $I$ with an interval in $\mathbf{R}$, then to each $g_{i}$ there corresponds a translation of $\mathbf{R}$ which agrees with $g_{i}$ on $I_{i}$. The maps $g_{i} \mid I_{i}$ constitute an interval exchange $A=A_{I}$ on I, whose domain and range consist of the complement of a finite set in $I$.

Now if $\phi$ is not an isometry, there are intervals $I$ and $I^{\prime}$ in $R$ that meet exactly in a common endpoint but are mapped isometrically onto the same interval $J$ in $T$. The interval exchanges $A_{I}$ and $A_{I^{\prime}}$ are conjugated by $\phi \mid I$ and $\phi \mid I^{\prime}$ to interval exchanges $B$ and $B^{\prime}$ on $J$. Since a composition of interval exchanges is an interval exchange, any positive word in $B$ and $B^{\prime}$ defines an interval exchange on $J$. One now fixes a generic point $z \in J$. Then $z$ is in the domain of every positive word in $B$ and $B^{\prime}$, and the restriction of each positive word to a small neighborhood of $z$ in $J$ coincides both with a translation and with an element of $\pi_{1} F$. It is possible to show that for each positive integer $N$, the $2^{N}$ positive words of length $N$ in $B$ and $B^{\prime}$ give rise to $2^{N}$ distinct elements of $\pi_{1} F$. On the other hand, since composition of translations is commutative, these $2^{N}$ positive words give rise to at most $N^{n+m}$ translations. Using this it is easy to produce a collection $\mathcal{D}_{n}$ of elements of $\pi_{1} F$ whose cardinality grows exponentially with $N$, such that each element of $\mathcal{D}_{N}$ fixes some neighborhood of $z$ in $J$, and such that maximum word length of the elements in $\mathcal{D}_{N}$, in terms of a fixed generating set for $\pi_{1} F$, grows linearly with $N$. This leads to a contradiction to the hypothesis that the action is small.

Thurston's theory of train tracks is used in this proof in an essential way. We consider a train track in $F$ carrying the lamination $\mathcal{L}$. In the argument sketched above, the elements of $\pi_{1} F$ defined by restricting positive words in $B$ and $B^{\prime}$ to a neighborhood of $z$ are all shown to be represented by smooth (based) loops in the train track. A key result, proved below as Proposition 2.2, asserts that smooth loops in a train track which are inequivalent, i.e. do not differ merely by reparameterization, define distinct elements of $\pi_{1} F$. This is used to show that the $2^{N}$ positive words of length $N$ in $B$ and $B^{\prime}$ give rise to $2^{N}$ distinct elements of $\pi_{1} F$.

The paper is organized as follows. In Sections 1 and 2 we review the definitions of trees and measured geodesic lamination. Also the above-mentioned result of Morgan-Otal and Hatcher, the theory of train tracks, and interval exchanges are discussed. 
Section 3 contains the proofs of the main theorem. There is an application to the theory of degenerations of hyperbolic structures on surfaces in Section 4. It is proved that the set of ideal points of $\mathcal{D}_{n}\left(\pi_{1} F\right)$ is canonically identified with the space of projective $\mathbf{R}$-measured geodesic laminations on $F$. In the case $n=2$ this was first proved by Thurston.

There were many people who contributed to the completion of this work. My first exposure to R-trees was from the lectures by John Morgan at the CBMS conference at the University of California at Los Angeles in 1986. I thank Mladen Bestvina and Mark Feighn for sharing their ideas on this problem. I am grateful to Chih-Han Sah for introducing me to the connection between measured laminations and interval exchanges and Ralf Spatzier for much technical advice. Also Marc Culler and Peter B. Shalen are responsible for many simplifications in the proofs. I acknowledge the support of Indiana University at Bloomington where I was visiting while part of this work was completed. Finally I thank the referee for his extensive revisions which were incorporated verbatim into this manuscript.

\section{Definitions}

Let $X$ be a metric space. A (closed) segment in $X$ is an arc isometric to a (closed) interval in $\mathbf{R}$.

An $\mathbf{R}$-tree is a non-empty, metric space $(T, d)$ satisfying

(i) any two points in $T$ are joined by a unique closed segment;

(ii) if two closed segments have a common endpoint, then their intersection is a closed segment; and

(iii) if two closed segments meet in exactly one common endpoint, then their union is a closed segment.

According to [Mo-Sh1, Theorem II.1.13], this definition is equivalent to the one given in the introduction. We shall sometimes refer to an $\mathbf{R}$-tree simply as a tree.

Given an $\mathbf{R}$-tree $T$ and $x \in T$, define $B_{x}=\{[x, y] \mid y \in T-\{x\}\}$. Define an equivalence relation by $[x, y] \sim[x, z]$ if $[x, y] \cap[x, z]=[x, w]$, for some $w \in T-\{x\}$. A direction at $x$ is an equivalence class in $B_{x}$. If $x$ has exactly two directions, then say $x$ is an edge, otherwise $x$ is a vertex. Notice that $x$ is an edge if and only if $T-\{x\}$ has exactly two connected components.

A morphism from an $\mathbf{R}$-tree $T$ to an $\mathbf{R}$-tree $T^{\prime}$ is a map $\phi: T \rightarrow T^{\prime}$, such that each non-degenerate segment may be written as a union of segments $\left[x_{0}, x_{1}\right],\left[x_{1}, x_{2}\right]$, $\ldots,\left[x_{n-1}, x_{n}\right]$, where $\phi \mid\left[x_{i-1}, x_{i}\right]$ is an isometric embedding. This definition is equivalent to the condition that for each non-degenerate segment $[x, y]$ there is a non-degenerate segment $[x, w] \subseteq[x, y]$ such that $\phi \mid[x, w]$ is an isometry. The morphism $\phi: T \rightarrow T^{\prime}$ is said to fold at a point $x \in T$ if there exist points $y, y^{\prime} \in T$ such that the segments $[x, y]$ and $\left[x, y^{\prime}\right]$ are non-degenerate and intersect only in $\{x\}$, and are mappped isometically by $\phi$ onto the same segment in $T^{\prime}$. It is easy to show that a morphism $\phi: T \rightarrow T^{\prime}$ which does not fold at any point of $T$ is an isometry onto a subtree of $T^{\prime}$.

By a group action on an $\mathbf{R}$-tree we mean a left action by isometries. We will often informally denote a given action of a group $\Gamma$ on a tree $T$ by $\Gamma \times T \rightarrow T$ when no confusion can arise. The action $\Gamma \times T \rightarrow T$ is said to be minimal if $T$ has no $\Gamma$-invariant proper subtree. It is shown in $[\mathrm{Al}-\mathrm{Ba}]$ and in $[\mathrm{Cu}-\mathrm{Mo}]$ that for every action $\Gamma \times T \rightarrow T$ there is a $\Gamma$-invariant subtree $T_{0}$ of $T$ such that the restricted 
action $\Gamma \times T_{0} \rightarrow T_{0}$ is minimal. In this paper it will be understood that all the actions that we consider are minimal, except when we specify to the contrary.

\section{Measured laminations}

In this section we review known facts about $\Lambda$-measured laminations on surfaces. Let $F$ be a hyperbolic surface of finite area. A geodesic lamination $\mathcal{L}$ is a closed subset of $F$, such that each path component is a simple geodesic. Let $\mathbf{H}^{2} \rightarrow F$ be the universal covering. A measured geodesic lamination $(\mathcal{L}, \mu)$ in $F$ has a preimage $(\tilde{\mathcal{L}}, \tilde{\mu})$ in $\mathbf{H}^{2}$. A transverse arc is essential, if some lift (hence every lift) meets each leaf of $\tilde{\mathcal{L}}$ at most once.

A transverse measure (or simply measure) $\mu$ is a function defined on the set of paths transverse to $\mathcal{L}$ and taking values in $\mathbf{R}$, such that

(i) $\mu\left(\gamma \gamma^{\prime}\right)=\mu(\gamma)+\mu\left(\gamma^{\prime}\right)$, whenever $\gamma, \gamma^{\prime}$ are transverse and the product is defined; and

(ii) $\mu(\gamma)=\mu\left(\gamma^{\prime}\right)$, whenever $\gamma, \gamma^{\prime}$ are transverse and are homotopic through a 1-parameter family of transverse paths.

It is understood that the support of $\mu$ is all of $\mathcal{L}$.

We shall say the action on a tree $\pi_{1} F \times T \rightarrow T$ is dual to the measured geodesic lamination $(\mathcal{L}, \mu)$ if there is an equivariant bijection $p$ from the connected components of $\mathbf{H}^{2}-\tilde{\mathcal{L}}$ to the vertices of $T$, such that $d(p(x), p(y))=\tilde{\mu}(\gamma)$, where $\gamma:[0,1] \rightarrow \mathbf{H}^{2}$ is a transverse arc from $x$ to $y$ meeting each leaf of $\tilde{\mathcal{L}}$ at most once. It is proved in [Mo-Sh4] that every measured geodesic lamination is dual to an action on an $\mathbf{R}$-tree, which is clearly unique up to equivariant isometry. It is also clear that the dual action is small.

We now review the theory of train tracks (see [Th1] or [Pe-Ha]). A smooth graph is an embedded graph $\tau \subseteq F$, such that for each point $p \in \tau$, there is a smooth open arc in $\tau$ through $p$ and any two such arcs are tangent at $p$. If $\mathcal{C}$ is an open subset of $F$ with compact closure, and if the frontier of $\mathcal{C}$ is contained in the smooth graph $\tau$, then $\mathcal{C}$ can be identified in a natural way with the interior of a compact manifoldwith-corners $\hat{\mathcal{C}}$. (If we give $\mathcal{C}$ the riemannian metric inherited from the hyperbolic metric on $F$, we may define $\hat{\mathcal{C}}$ to be the metric-space completion of $\mathcal{C}$.) We define the smooth Euler characteristic $\chi_{\text {smooth }}(\mathcal{C})$ to be $\chi_{\text {top }}(\mathcal{C})-n(\mathcal{C})$, where $\chi_{\text {top }}(\mathcal{C})$ denotes the topological Euler characteristic of $\mathcal{C}$ and $n(\mathcal{C})$ denotes the number of corners of $\hat{\mathcal{C}}$. It is straightforward to show that for any open subset $\mathcal{C}$ of $F$ whose frontier is contained in $\tau$, we have $\chi_{\text {smooth }}(\mathcal{C})=\sum_{C} \chi_{\text {smooth }}(C)$, where $C$ ranges over the components of $\mathcal{C}-\tau$. We define a train track in $F$ to be a smooth graph $\tau \subset F$ such that $\chi_{\text {smooth }}(C)<0$ for every component $C$ of $F-\tau$.

A train track $\tau$ carries a lamination $\mathcal{L}$ if there is a map $r: F \rightarrow F$ homotopic to the identity, such that $r \mid \alpha: \alpha \rightarrow \tau$ is a smooth immersion for each smooth arc $\alpha \subseteq \mathcal{L}$. The map $r$ is called a supporting map. Every geodesic lamination is carried by a (non-unique) train track ([Th1] or [Pe-Ha, Theorem 1.6.5]).

In Section 3 we will need a result, Proposition 2.2 below, concerning smooth loops in train tracks. Let $\tau$ be a smooth graph in a surface $F$, and let $\gamma:[0,1] \rightarrow \tau$ be a loop based at a point $* \in \tau$. We shall term $\gamma$ a smooth loop if the corresponding map $\gamma: S^{1} \rightarrow \tau \subset F$ is a smooth immersion of $S^{1}$ in $F$. If $*$ is not a vertex of $\tau$, the counterclockwise orientation of $S^{1}$ at the base point 1 gives rise to a local orientation determined by $\gamma$. 
Two smooth loops $\gamma, \gamma^{\prime}$ based at $* \in \tau$ will be termed equivalent if $\gamma^{\prime}=\gamma \circ h$ for some orientation-preserving diffeomorphism $h:[0,1] \rightarrow[0,1]$.

Lemma 2.1. Let $\tau$ be a train track in a surface $F$, and let $\gamma$ be a smooth loop in $\tau$. Then a lift $\tilde{\gamma}$ of $\gamma$ to the universal covering surface $\mathbf{H}^{2}$ of $F$ is an embedding of $[0,1]$ in $\mathbf{H}^{2}$.

Proof. If $\tilde{\gamma}$ is not an embedding, then there exist $s<t$ in $[0,1]$ such that $\tilde{\gamma}(s)=\tilde{\gamma}(t)$ and $\tilde{\gamma} \mid(s, t)$ is one-to-one. Since $\mathbf{H}^{2}$ is simply connected, the Jordan curve $\tilde{\gamma}([s, t])$ bounds an open disk $\mathcal{C} \subset \mathbf{H}^{2}$. The manifold-with-corners $\hat{\mathcal{C}}$ is a closed disk with at most one corner, and hence $\chi_{\text {smooth }}(\mathcal{C})>0$. But $\chi_{\text {smooth }}(\mathcal{C})=\sum_{C} \chi_{\text {smooth }}(C)$, where $C$ ranges over the components of $\mathcal{C}-\tau$, and the terms in this sum are negative according to the definition of a train track.

Proposition 2.2. Let $\tau$ be a train track in a surface $F$, let $*$ be a base point in $\tau$, and let $\gamma$ and $\gamma^{\prime}$ be inequivalent smooth loops in $\tau$ based at $*$. Then $\gamma$ and $\gamma^{\prime}$ represent distinct elements of $\pi_{1} F$.

Proof. Let $\tilde{*}$ be a base point in the universal covering surface $\mathbf{H}^{2}$ lying above $*$. Let $\tilde{\gamma}$ and $\tilde{\gamma}^{\prime}$ denote the lifts of $\gamma$ and $\gamma^{\prime}$ having initial point $\tilde{*}$. We must show that the terminal points $\tilde{\gamma}(1)$ and $\tilde{\gamma}^{\prime}(1)$ are distinct. Suppose to the contrary that $\tilde{\gamma}(1)=\tilde{\gamma}^{\prime}(1)$. By Lemma 2.1, $\tilde{\gamma}$ and $\tilde{\gamma}^{\prime}$ are embeddings, so that $\tilde{\gamma}([0,1])$ and $\tilde{\gamma}^{\prime}([0,1])$ are smooth arcs in $\mathbf{H}^{2}$, which by our assumption have the same end points. Since $\gamma$ and $\gamma^{\prime}$ are inequivalent, the arcs $\tilde{\gamma}([0,1])$ and $\tilde{\gamma}^{\prime}([0,1])$ are distinct. Hence there are subarcs $\tilde{\delta} \subset \tilde{\gamma}$ and $\tilde{\delta}^{\prime} \subset \tilde{\gamma}^{\prime}$ such that $\partial \tilde{\delta}=\partial \tilde{\delta}^{\prime}$ and int $\tilde{\delta} \cap$ int $\tilde{\delta}^{\prime}=\emptyset$. The Jordan curve $\tilde{\delta} \cup \tilde{\delta}^{\prime}$ bounds an open disk $\mathcal{C} \subset \mathbf{H}^{2}$. The manifold-with-corners $\tilde{\mathcal{C}}$ is a closed disk with at most two corners, and hence $\chi_{\operatorname{smooth}}(\mathcal{C}) \geq 0$. But $\chi_{\text {smooth }}(\mathcal{C})=\sum_{C} \chi_{\text {smooth }}(C)$, where $C$ ranges over the components of $\mathcal{C}-\tau$, and the terms in this sum are negative according to the definition of a train track.

The theory of train tracks can be used to prove the following result, which is the starting point for the proof of our main theorem. The result is essentially equivalent to a special case of the main result of Morgan and Otal's paper [Mo-Ot]. However, because geodesic laminations (and train tracks) are not discussed in [Mo-Ot], it is formally easier to deduce the theorem from the arguments in Hatcher's paper [Ha].

Theorem 2.3 (Morgan-Otal). Let $F$ be a hyperbolic surface of finite area and let $\pi_{1} F \times T \rightarrow T$ be an action on an $\mathbf{R}$-tree. Then there exist an action on an $\mathbf{R}$-tree $\pi_{1} F \times R \rightarrow R$ and a $\pi_{1} F$-equivariant morphism $\phi: R \rightarrow T$, such that $\pi_{1} F \times R \rightarrow R$ is dual to a measured geodesic lamination $(\mathcal{L}, \mu)$.

Furthermore, $\phi$ does not fold at an edge point; and if $\pi_{1} E$ fixes a point for each cusp $E$ of $F$, then $\mathcal{L}$ is compact.

Proof. It is shown on p. 86 of [Ha] that there exists an equivariant map $\tilde{f}: \mathbf{H}^{2} \rightarrow T$ which is transverse in the sense of [Mo-Sh3], and thus defines a topological measured lamination $\left(\mathcal{L}_{0}, \mu_{0}\right)$ on $\mathbf{H}^{2}$. According to Proposition 3.3 of [Ha], the map $\tilde{f}$ may be chosen so that $\left(\mathcal{L}_{0}, \mu_{0}\right)$ is carried by some train track $\tau$ in the sense defined above. (Hatcher uses the term "train track" to mean what we call a smooth graph, and uses "good train track" to mean a train track in our sense.) It now follows from the first two paragraphs on p. 85 and the last paragraphs on p. 87 of [Ha] that there exist an action $\pi_{1} F \times R_{0} \rightarrow R_{0}$ on an $\mathbf{R}$-tree, dual to $\left(\mathcal{L}_{0}, \mu_{0}\right)$, and an equivariant 
morphism $\phi_{0}: R_{0} \rightarrow T$. The transversality of the map $\tilde{f}$ implies that $\phi_{0}$ does not fold at an edge point.

It follows from [Pe-Ha, Theorem 2.7.4] that $\tau$ carries some geodesic measured lamination $(\mathcal{L}, \mu)$ with the same weights as $\left(\mathcal{L}_{0}, \mu_{0}\right)$. This implies that if $\pi_{1} F \times R \rightarrow$ $\mathbf{R}$ is an action dual to $(\mathcal{L}, \mu)$, then $\pi_{1} F \times R \rightarrow R$ has the same length function (in the sense of [Mo-Sh1], [Al-Ba] and [Cu-Mo]) as $\pi_{1} F \times R_{0} \rightarrow R_{0}$. (Indeed, the length of any element $g \in \pi_{1} F$ with respect to the action on $R$ or $R_{0}$ is the minimum of $\mu(\gamma)$ or $\mu_{0}(\gamma)$, where $\gamma$ ranges over all closed curves representing the conjugacy class of $g$. If $g$ is given, this minimum can be computed in terms of the weights of $(\mathcal{L}, \mu)$ or $\left(\mathcal{L}_{0}, \mu_{0}\right)$.) It now follows from [Al-Ba] or [Cu-Mo] that the minimal $\pi_{1} F$-invariant subtree of $R_{0}$ is $\pi_{1} F$-equivariantly isometric to $R$ (which is its own minimal $\pi_{1} F$-invariant subtree). The required $\pi_{1} F$-equivariant morphism $\pi: R \rightarrow T$ can now be obtained by pre-composing $\phi_{0}$ with an equivariant isometry of $R$ onto the minimal $\pi_{1} F$-invariant subtree of $R_{0}$.

If $\pi_{1} E$ fixes a point of $E$ for each cusp $E$ of $F$, it is easy to modify the construction of $\phi_{0}$ so as to guarantee that $\phi_{0}$ is locally constant on a neighborhood of $\partial F$. This implies that $\mathcal{L}_{0}$ is compact and is carried by a compact train track $\tau$, and hence $\mathcal{L}$ is compact as well.

Finally we review how a measured compact geodesic lamination determines an interval exchange. Let $I$ be a closed, finite interval, and let $I_{1}, \ldots, I_{n} \subseteq I$ be pairwise disjoint open intervals whose closures cover $I$. Then an interval exchange on $I$ is a map $A: \bigcup I_{i} \rightarrow I$ such that $A \mid I_{i}$ is a translation; and $A\left(I_{1}\right), \ldots, A\left(I_{n}\right)$ are pairwise disjoint. Notice each power of $A$ is defined at all but finitely many points of $I$.

Fix a measured compact geodesic lamination $(\mathcal{L}, \mu)$. Let $\alpha$ be an arc transverse to $\mathcal{L}$ with $\alpha \cap \mathcal{L} \neq \emptyset$. Fix both an orientation and a transverse orientation on $\alpha$.

For each point $u \in \alpha \cap \mathcal{L}$ there is a unique oriented arc $\gamma_{u}$ contained in a leaf of $\mathcal{L}$ such that

(i) the initial point of $\gamma_{u}$ is $u$ and its terminal point lies on $\alpha$;

(ii) the orientation of $\gamma_{u}$ at its initial and terminal points agrees with the transverse orientation of $\alpha$; and

(iii) no non-degenerate subarc of $\gamma_{u}$ satisfies (i) and (ii).

Furthermore, there are finitely many disjoint open subintervals $\alpha_{1}, \ldots, \alpha_{n} \subset \alpha$ covering $\alpha \cap \mathcal{L}$ such that for each $i \in\{1, \ldots, n\}$, the oriented arcs $\gamma_{u}$ for $u \in \alpha_{i} \cap \mathcal{L}$ form the product family $\mathcal{F}_{i}$. The terminal points of the arcs in the family $\mathcal{F}_{i}$ constitute a set of the form $\beta_{i} \cap \mathcal{L}$ where $\beta_{i}$ is an open subinterval of $\alpha$. The $\beta_{i}$ cover $\alpha \cap \mathcal{L}$, and they may be taken to be disjoint. We have $\mu\left(\beta_{i}\right)=\mu\left(\alpha_{i}\right)$.

Now let $I$ be an oriented interval of length $\mu(\alpha)$. There are uniquely determined disjoint open subintervals $I_{1}, \ldots, I_{n}$ in $I$ which are ordered in $I$ in the same way that the $\alpha_{i}$ are ordered in $\alpha$ and such that each $I_{i}$ has length $\mu\left(\alpha_{i}\right)$. Similarly, there are uniquely determined disjoint open subintervals $J_{1}, \ldots, J_{n}$ in $I$ which are ordered in $I$ in the same way that the $\beta_{i}$ are ordered in $\alpha$ and such that each $I_{i}$ has length $\mu\left(\beta_{i}\right)=\mu\left(\alpha_{i}\right)$. Let $A: \bigcup I_{i} \rightarrow I$ be the unique interval exchange such that $A\left(I_{i}\right)=J_{i}$ for each $i=1, \ldots, n$.

Each point $u \in \alpha \cap \mathcal{L}$ determines a (possibly degenerate) closed subinterval $\eta_{u}=\left[h_{u}^{-}, h_{u}^{+}\right]$of $I$ : here $h_{u}^{-}=\mu\left(\alpha_{u}^{-}\right)$, where $\alpha_{u}^{-}$is the open subinterval of $\alpha$ bounded by $u$ and the initial point of $\alpha$, and $h_{u}^{+}=\mu\left(\alpha_{u}^{+}\right)$, where $\alpha_{u}^{+}$is the open subinterval of $\alpha$ bounded by $u$ and the terminal point of $\alpha$. For any $u$ we have 
$A\left(\eta_{u}\right)=\eta_{v}$, where $v$ denotes the terminal point of $\gamma_{u}$. In fact $A \mid \eta_{u}$ is the unique translation mapping $\eta_{u}$ onto $\eta_{v}$. This characterizes the interval exchange $A$ directly in terms of the measured lamination $(\mathcal{L}, \mu)$ and the oriented, transversely oriented transverse $\operatorname{arc} \alpha$.

If $\tau$ is a train track carrying a given measured lamination $(\mathcal{L}, \mu)$, with supporting map $r$, and if $\alpha \subset F$ is any arc such that $r(\alpha)$ is a single point of $\tau$ which is not a vertex, then $\alpha$ is transverse to $\mathcal{L}$. In this case we may choose the $\operatorname{arcs} \alpha_{i}$ in the above construction in such a way that for each $i \in\{1, \ldots, n\}$, the arcs in the parallel family $\mathcal{F}_{i}$ are all projected by $r$ to mutually equivalent smooth loops in $\tau$, based at $*=r(\alpha)$. Thus the $\mathcal{F}_{i}$ determine smooth loops $\gamma_{1}, \ldots, \gamma_{n}$ that are well-defined up to equivalence. In particular they determine elements $\left[\gamma_{1}\right], \ldots,\left[\gamma_{n}\right]$ of $\pi_{1} F=\pi_{1}(F, *)$.

Finally, we point out how the interval exchange $A$ is related to the dual tree to $(\mathcal{L}, \mu)$. For this purpose we choose a base point $\tilde{*} \in \mathbf{H}^{2}$ that projects to $*$. The arc $\alpha$ has a unique lift $\tilde{\alpha}$ containing $\tilde{*}$. Let us use $\tilde{*}$ to identify $\pi_{1} F$ with the group of covering transformations in $\mathbf{H}^{2}$. Let $\pi_{1} F \times T \rightarrow T$ denote an action of $\pi_{1} F$ on an $\mathbf{R}$-tree which is dual to $(\mathcal{L}, \mu)$, and let $p: \mathbf{H}^{2} \rightarrow T$ be the $\pi_{1} F$-equivariant map appearing in the definition of a dual action. Then $p(\tilde{\alpha})$ is a segment $[x, y]$ of length $\mu(\alpha)$ : here $x$ and $y$ respectively denote the images of the initial and terminal points of $\tilde{\alpha}$. Let us identify $[x, y]$ isometrically with the oriented interval $I$ in such a way that $x$ becomes the initial point. Then $A$ becomes an interval exchange on $[x, y]$. The above characterization of $A$ implies that for $i=1, \ldots, n$ we have $A\left|I_{i}=\left[\gamma_{i}\right]\right| I_{i}$. This description of the interval exchange in terms of the dual tree will be the starting point for the proof of our main theorem in the next section.

\section{THE MAIN THEOREM}

In this section we prove the main theorem. The proof of Proposition 3.1 contains all the hard work. It depends on three lemmas. The proof makes strong use of Proposition 2.2. Some of the ideas in the proof are derived from arguments used in [Pla], [Mo-Sh2], [Mo2] and [Mo-Sk].

Proposition 3.1. Let $F$ be a hyperbolic surface of finite area and let $\pi_{1} F \times R \rightarrow R$ be an action on an $\mathbf{R}$-tree which is dual to a measured compact geodesic lamination. Let $\pi_{1} F \times T \rightarrow T$ be an action on an $\mathbf{R}$-tree and let $\phi: R \rightarrow T$ be a $\pi_{1} F$-equivariant morphism. If $\phi$ folds at some vertex point, then the action on $T$ is not small.

Proof. Suppose $\phi$ folds at the vertex $x \in R$, so there are non-degenerate segments $[x, y]$ and $\left[x, y^{\prime}\right]$ in $T$ such that $[x, y] \cap\left[x, y^{\prime}\right]=\{x\} ; \phi|[x, y], \phi|\left[x, y^{\prime}\right]$ are embeddings; and $\phi([x, y])=\phi\left(\left[x, y^{\prime}\right]\right)$. Let $\pi_{1} F \times R \rightarrow R$ be dual to the measured compact geodesic lamination $(\mathcal{L}, \mu)$ with corresponding map $p: \mathbf{H}^{2}-\tilde{\mathcal{L}} \rightarrow R$. There is some component $\tilde{C}$ of $\mathbf{H}^{2}-\tilde{\mathcal{L}}$, such that $p(\tilde{C})=x$.

There is an essential arc $\tilde{\alpha}$ whose end points are mapped by $p$ to $x$ and $y$. Similarly, there is an essential arc $\tilde{\alpha}^{\prime}$ whose end points are mapped to $x$ and $y^{\prime}$. The arcs $\tilde{\alpha}$ and $\tilde{\alpha}^{\prime}$ meet in a single point $\tilde{*} \in \tilde{C}$ which we regard as a base point for $\mathbf{H}^{2}$. The image of $*$ in $F$ will be regarded as a base point for $F$. After possibly replacing $[x, y],\left[x, y^{\prime}\right]$ and $\tilde{\alpha}, \tilde{\alpha}^{\prime}$ by shorter segments and arcs, respectively, suppose that $\tilde{\alpha} \cup \tilde{\alpha}^{\prime}$ is disjoint from its translates. It follows that $\tilde{\alpha} \cup \tilde{\alpha}^{\prime}$ projects to an embedded arc $\alpha \cup \alpha^{\prime}$ in $F$. 
Now $\mathcal{L}$ is carried by some train track $\tau$ with supporting map $r$. After possibly rechoosing the train track and $r$, assume $r\left(\alpha \cup \alpha^{\prime}\right)$ is a single point which is not a vertex of the train track.

Choose an orientation and a transverse orientation on $\alpha \cup \alpha^{\prime}$. According to the discussion at the end of Section 2, we can associate to the oriented, transversely oriented, $\mathcal{L}$-transverse arc $\alpha$ and oriented interval $I$, an interval exchange $A: \bigcup_{i=1}^{n} I_{i} \rightarrow I$ and smooth loops $\gamma_{1}, \ldots, \gamma_{n}$ in $\tau$, based at $*=r\left(\alpha \cup \alpha^{\prime}\right)$ (and welldefinied up to equivalence). In the same way we can associate to $\alpha^{\prime}$ and oriented interval $I^{\prime}$, an interval exchange $A^{\prime}: \bigcup_{i=1}^{n} I_{i}^{\prime} \rightarrow I^{\prime}$ and smooth loops $\gamma_{1}^{\prime}, \ldots, \gamma_{n}^{\prime}$ in $\tau$, based at $r\left(\alpha \cup \alpha^{\prime}\right)$.

The $\gamma_{i}$ and $\gamma_{j}^{\prime}$ all determine the same local orientation of $\tau$ at $x$ because they are all defined using a fixed transverse orientation of $\alpha \cup \alpha^{\prime}$. It is also clear from the constructions in Section 2 that the $\gamma_{i}$ and the $\gamma_{j}^{\prime}$ are all minimal smooth loops in the sense that none of them has a non-degenerate proper subpath which is a smooth loop based at $*$.

Since $\alpha$ and $\alpha^{\prime}$ correspond to distinct boundary components of $\tilde{C}$, no $\gamma_{i}$ is equivalent to any $\gamma_{j}^{\prime}$.

The $\gamma_{i}$ and $\gamma_{j}^{\prime}$ represent elements $\left[\gamma_{i}\right]$ and $\left[\gamma_{j}^{\prime}\right]$ of $\pi_{1}(F, *)$. Using the base point $\tilde{*}$ to identify $\pi_{1}(F, *)$ with the group of covering transformations in $\mathbf{H}^{2}$, we may regard $\left[\gamma_{i}\right]$ and $\left[\gamma_{j}^{\prime}\right]$ as covering transformations. Let us also identify $I$ and $I^{\prime}$ with $[x, y]$ and $\left[x^{\prime}, y^{\prime}\right]$ respectively, in such a way that $x$ and $x^{\prime}$ become the initial points. According to the final paragraph of Section 2 we have $A\left|I_{i}=\left[\gamma_{i}\right]\right| I_{i}$ for all $i=1, \ldots, n$ and $A^{\prime}\left|I_{j}^{\prime}=\left[\gamma_{j}\right]\right| I_{j}$ for all $j=1, \ldots, m$.

Now let $J$ denote the segment $\phi([x, y])=\phi\left(\left[x, y^{\prime}\right]\right)$. Set $J_{i}=\phi\left(I_{i}\right)$ for $i=$ $1, \ldots, n$ and $J_{j}^{\prime}=\phi\left(I_{j}^{\prime}\right)$ for $j=1, \ldots, m$. Conjugating the interval exchanges $A$ and $A^{\prime}$ by the isometries $\phi \mid[x, y]$ and $\phi \mid\left[x, y^{\prime}\right]$, we obtain two interval exchanges $B$ and $B^{\prime}$ on $J$. In view of the equivalence of $\phi$ we have:

$$
\begin{aligned}
B \mid J_{i} & =\left[\gamma_{i}\right] \mid J_{i} \quad \text { for } i=1, \ldots, n, \text { and } \\
B^{\prime} \mid J_{j}^{\prime} & =\left[\gamma_{j}^{\prime}\right] \mid J_{j}^{\prime} \quad \text { for } j=1, \ldots, m .
\end{aligned}
$$

Let $\Theta$ denote the semigroup generated by $B$ and $B^{\prime}$. We fix a point $z \in J$ such that all elements of $\Theta$ are defined at $z$. For each positive integer $N$ we set $\mathcal{C}_{N}=\{0,1\}^{N}$; thus $\mathcal{C}_{N}$ has cardinality $2^{N}$. For each $c=\left\langle\epsilon_{1}, \ldots, \epsilon_{N}\right\rangle \in \mathcal{C}_{N}$ we define an element $\theta_{c} \in \Theta$ by setting $\theta_{c}=B_{N} \circ \cdots \circ B_{1}$, where $B_{p}=B$ whenever $\epsilon=0$, and $\mathcal{B}_{p}=B^{\prime}$ whenever $\epsilon=1$. Since the domain of $B$ consists of $n$ intervals on each of which $B$ restricts to a translation, and the domain of $B^{\prime}$ consists of $m$ intervals on each of which $B^{\prime}$ restricts to a translation, and since composition of translations is commutative, the set $\left\{\theta_{c}(z): c \in \mathcal{C}_{N}\right\}$ has cardinality at most $N^{n+m}$.

There is a natural way of associating to each $c \in \mathcal{C}_{N}$ an $N$-tuple of smooth loops $\left\langle\eta_{1}, \ldots, \eta_{N}\right\rangle$ in $\tau$. To do this we write $c=\left\langle\epsilon_{1}, \ldots, \epsilon_{N}\right\rangle \in \mathcal{C}_{N}$ and define $B_{1}, \ldots, B_{N}$ as in the above definition of $\theta_{c}$. If $p \in\{1, \ldots, N\}$ is an index such that $\epsilon_{p}=0$ and if $i \in\{1, \ldots, n\}$ denotes the index such that $B_{p-1} \circ \cdots \circ B_{1}(z) \in J_{i}$, we set $\eta_{p}=\gamma_{i}$. On the other hand, if $\epsilon_{p}=1$ and if $j \in\{1, \ldots, m\}$ denotes the index such that $B_{p-1} \circ \cdots \circ B_{1}(z) \in J_{j}^{\prime}$, we set $\eta_{p}=\gamma_{j}^{\prime}$. The composite loop $\omega_{c}=\eta_{N} * \cdots * \eta_{1}$ defines an element $\left[\omega_{c}\right]$ of $\pi_{1} F$, which we identify with a covering transformation. It follows from the observation $(*)$ above that $\left[\omega_{c}\right](z)=\theta_{c}(z)$, and indeed that $\left[\omega_{c}\right]$ and $\theta_{c}$ agree on a neighborhood of $z \in J$. 
Since the $\gamma_{i}$ and the $\gamma_{j}^{\prime}$ all determine the same local orientation of $\tau$ and $*$, the loop $\omega_{c}$ is smooth for every $c \in \mathcal{C}_{N}$. Since the $\gamma_{i}$ and $\gamma_{j}^{\prime}$ are all minimal smooth loops and since no $\gamma_{i}$ is equivalent to any $\gamma_{j}^{\prime}$, the smooth loops $\omega_{c}$ and $\omega_{c^{\prime}}$ are inequivalent whenever $c$ and $c^{\prime}$ are distinct elements of $\mathcal{C}_{N}$. It therefore follows from Proposition 2.2 that the $2^{N}$ elements $c \in \mathcal{C}_{N}$ determine $2^{N}$ distinct elements $\left[\omega_{c}\right]$ of $\pi_{1} F$.

On the other hand, the set $\left\{\theta_{c}(z) \mid c \in \mathcal{C}_{N}\right\}=\left\{\left[\omega_{c}\right](z) \mid c \in \mathcal{C}_{N}\right\}$ has cardinality at most $N^{n+m}$. Hence there is a sequence $\left\langle\mathcal{D}_{N}\right\rangle_{N \geq 1}$ of subsets of $\pi_{1} F$ such that

(i) $\mathcal{D}_{N} \subset\left\{\left[\omega_{c}\right] \mid c \in \mathcal{C}_{N}\right\}$;

(ii) the cardinality of $\mathcal{D}_{N}$ grows exponentially as $N \rightarrow \infty$; and

(iii) for each $N$, the elements of $\mathcal{D}_{N}$, regarded as covering transformations, all agree at $z$.

For each $N$ set $\mathcal{E}_{N}=\mathcal{D}_{N}^{-1} \mathcal{D}_{N} \subset \pi_{1} F$. Then the cardinality of $\mathcal{E}_{N}$ grows exponentially as $N \rightarrow \infty$, and the $\mathcal{E}_{N}$ are all contained in the stabilizer of $z$. Moreover, since each element of $\mathcal{D}_{N}$ has the form $\left[\omega_{c}\right]$ and thus agrees with an interval exchange $\theta_{c}$ on some neighborhood of $z$ in $J$, each element of $\mathcal{E}_{N}$ actually fixes some neighborhood of $z$ in $J$. Hence any two elements of the set $\mathcal{E}=\bigcup_{N=1}^{\infty} \mathcal{E}_{N}$ have a common fixed segment in $T$. If the action of $\Gamma$ on $T$ is small, the stabilizer of every segment in $T$ is cyclic. This implies that the group generated by $\mathcal{E}$ is abelian-and therefore cyclic since it is contained in $\pi_{1} F$.

To complete the proof we use a simple corollary of Dehn's algorithm for the surface group $\pi_{1} F$ [L-S, Chapter V, Section 4], namely that any cyclic subgroup $Z$ of $\pi_{1} F$ has linear extrinsic growth. This means that if $S$ is a finite generating set for $\pi_{1} F$, then the number of elements of $Z$ that can be expressed as words of at most a given length $l$ in $S$ is bounded by a linear function of $l$. Note that if $K$ denotes the maximum length in a fixed generating set $S$ of elements $\gamma_{1}, \ldots, \gamma_{n}, \gamma_{1}^{\prime}, \ldots, \gamma_{m}^{\prime}$, then for each $N$ the elements of the set $\mathcal{E}_{N}$ are all of lengh at most $2 K N$. Since the cardinality of $\mathcal{E}_{N}$ grows exponentially with $N$, the linear extrinsic growth of the cyclic group generated by $\mathcal{E}$ is not cyclic, and so the action of $\pi_{1} F$ on $T$ is not small.

Here is our main result. In the following statement, $\pi_{1} E$ is identified via the inclusion homomorphism with a subgroup of $\pi_{1} F$ which is well-defined up to conjugation.

Theorem 3.2. Let $F$ be a hyperbolic surface of finite area and let $\pi_{1} F \times T \rightarrow T$ be a non-trivial action on an $\mathbf{R}$-tree, such that $\pi_{1} E$ fixes a point for each cusp $E$ of $F$. Then $\pi_{1} F \times T \rightarrow T$ is small if and only if it is dual to a measured compact geodesic lamination.

Proof. Suppose $\pi_{1} F \times T \rightarrow T$ is dual to a measured geodesic lamination $(\mathcal{L}, \mu)$. If an element of $\pi_{1} F$ fixes a segment in $T$, then it leaves invariant each component of the corresponding parallel family of leaves in $\tilde{\mathcal{L}}$. In particular, the stabilizer of a segment is contained in the stabilizer of some leaf and is necessarily cyclic.

Now suppose that the action is small. By Theorem 2.3 there exist an action $\pi_{1} F \times R \rightarrow R$ which is dual to a measured compact geodesic lamination $(\mathcal{L}, \mu)$ and a $\pi_{1} F$-equivariant morphism $\phi: R \rightarrow T$. Furthermore, $\phi$ does not fold at an edge point. Since the action on $T$ is small, Proposition 3.1 says $\phi$ does not fold at a vertex. Hense $\phi$ maps $R$ isometrically onto a subtree $T_{0}$ of $T$. Since $\phi$ is 
$\pi_{1} F$-equivariant and $\pi_{1} F \times T \rightarrow T$ is minimal, we must have $T_{0}=T$. This shows that $\pi_{1} F \times T \rightarrow T$ is dual to $(\mathcal{L}, \mu)$.

\section{Degenerations of hyperbolic STRUCTURES ON SURFACES}

If $\Gamma$ is a finitely generated group, we let $\mathcal{D}_{n}(\Gamma)$ denote the space of discrete, faithful representations of $\Gamma$ in the isometry group of hyperbolic $n$-space, modulo conjugation. In [Mo-Sh1], Morgan and Shalen construct a canonical compactification $\hat{\mathcal{D}}_{n}(\Gamma)$ of $\mathcal{D}_{n}(\Gamma)$. The "boundary" $\partial \mathcal{D}_{n}(\Gamma)=\hat{\mathcal{D}}_{n}(\Gamma)-\mathcal{D}_{n}(\Gamma)$ is identified with a compact subset of the "projective space" $\mathcal{P}=\left([0, \infty)^{\Gamma}-\{0\}\right) / \mathbf{R}^{+}$: here $\mathbf{R}^{+}$acts on $[0, \infty)^{\Gamma}-\{0\}$ by conjugation, $[0, \infty)^{\Gamma}$ has the product topology, $[0, \infty)^{\Gamma}-\{0\}$ has the subspace topology, and the orbit space $\mathcal{P}$ is given the quotient topology.

Now let $F$ be a hyperbolic surface of finite area. Each measured lamination $(\mathcal{L}, \mu)$ on $F$ determines a length function $\ell: \pi_{F} \rightarrow \mathbf{R}$, where $\ell(G)$ is defined to be the intersection number of $\mathcal{L}$ of the closed geodesic representing the conjugacy class of $g$. We may regard $\ell$ as a point of $[0, \infty)^{\pi_{1} F}-\{0\}$, and its image in $\mathcal{P}$ is called the projectivized length function associated to $(\mathcal{L}, \mu)$. We denote by $\mathcal{P} \mathcal{M L}(F)$ the subset of $\mathcal{P}$ consisting of all projectivized length functions associated to measured laminations on $\mathcal{F}$.

The purpose of this section is to point out that the following theorem follows from Theorem 3.2 above together with the results of [Mo-Sh1]. The case $n=2$ was proved by Thurston (see [FLP]) and subsequent proofs were given in [Mo-Sh1], [Mo-Ot] and [Sk2].

Theorem 4.1. Let $F$ be a hyperbolic surface of finite area. For all $n \geq 2$ we have $\partial \mathcal{D}_{n}=\mathcal{P} \mathcal{M L}(F)$.

Proof. Recall from [Mo-Sh1, II.2.11] that every action $\Gamma \times T \rightarrow T$ of a group on an $\mathbf{R}$-tree determines a length function $\ell: \pi_{1} F \rightarrow \mathbf{R}$ defined by $\ell(g)=$ $\min _{x \in T} d(x, g \cdot x)$. If the action is non-trivial, that is, if it has no fixed point in $T$, then $\ell \neq 0$ by [Mo-Sh1, Proposition II.2.15]. We may therefore regard $\ell$ as an element of $[0, \infty)^{\pi_{1} F}-\{0\}$, and its image in $\mathcal{P}$ is called the projectivized length function associated to the action of $\Gamma$ on $T$. For any finitely generated group $\Gamma$ we denote by $\mathcal{P} \mathcal{S} \mathcal{L F}(F)$ the set of all projectivized length functions defined by non-trivial small actions of $\Gamma$ on R-trees. According to [Mo-Sh1, Theorem II.4.7], for any finitely generated group $\Gamma$ and any $n \geq 2$ we have $\partial \mathcal{D}_{n}(\Gamma) \subset \mathcal{P} \mathcal{L} \mathcal{F}(F)$. (The actions considered in [Mo-Sh1] are not necessarily minimal, but it is shown in $[\mathrm{Cu}-\mathrm{Mo}]$ and in $[\mathrm{Al}-\mathrm{Ba}]$ that a length function defined by a non-trivial action is also defined by a non-trivial small minimal action.)

When $\Gamma$ is the fundamental group of a finite-area hyperbolic surface $F$ and $\Gamma \times T \rightarrow T$ is the action dual to a compact measured lamination $(\mathcal{L}, \mu)$ on $F$, it is straightforward to check that the length function defined by the action coincides with the length function defined by $(\mathcal{L}, \mu)$ in the above sense. Hence it follows from Theorem 3.2 above that $\mathcal{P} \mathcal{S} \mathcal{L} \mathcal{F}\left(\pi_{1} F\right)=\mathcal{P} \mathcal{M L}(F)$. In particular we have $\partial \mathcal{D}_{n} \subset \mathcal{P} \mathcal{M L}(F)$. To prove the reverse inclusion, one uses the fact [Pe-Ha] that $\mathcal{P} \mathcal{M L}(F)$ has a dense subset consisting of rational points. By definition these arise from measured laminations of the form $(\mathcal{L}, \mu)$, where $\mathcal{L}$ is a disjoint union of simple closed curves and $\mu$ is integer-valued. But it is shown, for example, in [Mo-Sh1, Proposition III.3.2] that every rational point of $\mathcal{P} \mathcal{M L}(F)$ lies in $\partial \mathcal{D}_{2}$. Since $\partial \mathcal{D}_{2}$ is closed in $\mathcal{P}$ it follows that $\mathcal{P} \mathcal{M L}(F) \subset \partial \mathcal{D}_{2}$. Finally, it is obvious that $\partial \mathcal{D}_{2} \subset \partial \mathcal{D}_{n}$, so that $\partial \mathcal{D}_{2} \subset \partial \mathcal{D}_{n}$. 
It is natural to ask whether a proof of the main theorem of [Th3] can be based on Theorem 4.1 .

\section{REFERENCES}

[Al-Ba] R. Alperin and H. Bass, "Length Functions of Group Actions on $\Lambda$-Trees," in: S. M. Gersten et al., eds., Combinatorial Group Theory and Topology, Annals of Math Studies \#111, Princeton University Press, Princeton, (1987) 265-378. MR 89c:20057

[Ba] H. Bass, "Group Actions on Non-Archimedean Trees," in: R. C. Alperin, ed., Arborea Group Theory, Mathematical Sciences Research Institute Publications, \# 19, SpringerVerlag, New York (1991) 69-131. MR 93d:57003

[Cu-Mo] M. Culler and J. W. Morgan, "Group Actions on R-Trees," Proc. Lond. Math. Soc., 55, (1987) 571-604. MR 88f:20055

[FLP] A. Fathi, F. Laudenbach, V. Poenaru, "Travaux de Thurston sur les surfaces," Séminaire Orsay, Astérisque, 66-67 (1979) Soc. Math. de France, Paris. MR 82m:57003

[Gi-Sh] H. Gillet and P. B. Shalen, "Dendrology of Groups in low Q-ranks," J. Diff. Geo., 32 (1990) 605 - 712. MR 92b:57003

[Gi-Sh-Sk] H. Gillet, P. B. Shalen and R. K. Skora, "Simplicial Approximation and Low-rank Trees," Comment. Math. Helvetici, 66 (1991) 521-540. MR 93a:20038

[Ha] A. Hatcher, "Measured Lamination Spaces for Surfaces from the Topological Viewpoint," Top. Appl., 30, (1988), 63-88. MR 89k:57022

[Ji1] R. Jiang, "Branch Points and Free Actions on R-Trees," in: R. C. Alperin, ed., Arboreal Group Theory, Mathematical Sciences Research Institute Publications, \# 19, SpringerVerlag, New York, (1991) 251-293. MR 92b:57001

[Ji2] R. Jiang, "On Free Actions on R-Trees," Ph.D. Thesis, Columbia University, (1990)

[L-S] R. C. Lyndon and P. E. Schupp, Combinatorial Group Theory, Ergebnisse der Mathematik und ihrer Grenzgebiete, Bd. 89, Springer-Verlag, (1977). MR 58:28182

[Mo1] J. W. Morgan, "Group Actions on Trees and the Compactification of the Spaces of Classes of $S O(n, 1)$-representations," Top., 25 (1986) 1-34. MR 87h:20062

[Mo2] J. W. Morgan, "Ergodic Theory and Free Actions on Trees," Invent. Math. 94 (1988) 605-622. MR 90d:20069

[Mo-Ot] J. W. Morgan and J-P. Otal, "Relative Growth Rates of Closed Geodesics on a Surface under varying Hyperbolic Structures," (preprint).

[Mo-Sh1] J. W. Morgan and P. B. Shalen, "Degenerations of Hyperbolic Structures, I: Valuations, Trees and Surfaces," Ann. Math., 120 (1984) 401-476. MR 86f:57011

[Mo-Sh2] J. W. Morgan and P. B. Shalen, "Degenerations of Hyperbolic Structures, II: Measured Laminations in 3-Manifolds," Ann. of Math., 127 (1988) 403-456. MR 89e:57010a

[Mo-Sh3] J. W. Morgan and P. B. Shalen, "Degenerations of Hyperbolic Structures, III: Actions of 3-Manifold Groups on Trees and Thurston's Compactness Theorem," Ann. of Math., 127 (1988) 457-519. MR 89e:57010b

[Mo-Sh4] J. W. Morgan and P. B. Shalen, "Free Actions of Surface Groups on R-Trees," Topology, 30 (1991) 143 - 154. MR 92e:20016

[Mo-Sk] J. W. Morgan and R. K. Skora, "Groups Acting Freely on R-trees," Ergodic Theory and Dynamical Systems, 11 (1991) 737-756. MR 94a:20041

[Pe-Ha] R. Penner, with J. Harer, Combinatorics of Train Tracks, Annals of Mathematics Studies, \# 125, Princeton University Press, Princeton (1992). MR 94b:57018

[Pla] J. Plante, "Foliations with Measure preserving Holonomy," Ann. of Math. 102 (1975) 327-361. MR 52:11947

[Ser] J.-P. Serre, Trees, Springer-Verlag, New York (1980). MR 82c:20083

[Sh] P. B. Shalen, "Dendrology of Groups: An Introduction," in: S. M. Gersten ed., Essays in Group Theory, Mathematical Sciences Research Institute Publications, \#8, Springer-Verlag, New York (1987) 265-319. MR 89d:57012

[Sk1] R. K. Skora, "Research Announcement : Splittings of Surfaces," Bull. A.M.S., 23 (1990) 85-90. MR 90k:57016

[Sk2] R. K. Skora, "Geometric Actions of Surface Groups on $\Lambda$-Trees," Comment. Math. Helvetici, 65 (1990) 517-533. MR 91k:20035

[Sk3] R. K. Skora, "Combination Theorems for Actions on Trees," (preprint, 1989). 
[Sk4] R. K. Skora, "Deformations of Length Functions in Groups," (preprint, 1990).

[Th1] W. Thurston, "The Geometry and Topology of three-Manifolds," (preprint, 1979).

[Th2] W. Thurston, "Hyperbolic Structures on 3-Manifolds, I: Deformation of acylindrical manifolds," Ann. Math., 124 (1986) 203-246. MR 88g:57014

[Th3] W. Thurston, "Hyperbolic Structures on 3-Manifolds, II: Surface Groups and 3Manifolds which Fiber over the Circle," (preprint, 1986).

Abstract. The main result characterizes small actions of surface groups on R-trees.

Department of Mathematics, State University of New York, Stony Brook, New York 11794-3651

Department of Mathematics, Columbia University, New York, New York 10027-6918

E-mail address: rskora@interserv.com 